\title{
Effect of Seed Priming on Standard Germination and Electrical Conductivity in Marigold Seeds
}

\author{
K. Sindhu* and S.K. Sehrawat \\ Department of Horticulture- Floriculture and Landscape Architecture, CCS Haryana \\ Agricultural University, Hisar, India \\ *Corresponding author
}

\section{A B S T R A C T}

\section{Keywords}

Marigold, Seed, Priming, Standard germination, Electrical conductivity

\section{Article Info}

Accepted:

12 October 2019

Available Online:

10 November 2019
Marigold is one of the important annual flowers grown for commercial purpose all over the world. Non availability of high quality seeds of marigold is one of the major constraints in its cultivation. Priming of Marigold seeds plays crucial role in its germination and electrical conductivity, Hence a laboratory study was carried out to investigate the influence of various priming treatments on standard germination and electrical conductivity of French marigold seeds. This study was carried out on marigold seeds of four lines and nine treatments. Seeds treated with $0.5 \% \mathrm{KNO}_{3}$ exhibited significantly highest germination $(83.00 \%)$ during 0 month storage. In 2, 4, 6, 8 and 10 months storage duration, significantly maximum germination percentage was observed in $\mathrm{T}_{4}(81.33 \%, 75.33 \%, 69.33 \%, 60.91 \%$ and $44.25 \%)$ respectively. Maximum germination percentage was recorded in G-4 (83.55\%) which was followed by G-1 (79.33\%) and G-3 (76.22\%) during 0 month storage. In $2,4,6,8$ and 10 months storage duration, significantly maximum germination percentage was observed in G-4 $(81.741 \%, 74.44 \%$, $65.92 \%, 55.96 \%$ and $43.96 \%)$ respectively. The highest electrical conductivity was recorded in $\mathrm{T}_{0}(0.499 \mathrm{ds} / \mathrm{cm} / \mathrm{seed})$ during 0 month storage. In 2, 4, 6, 8 and 10 months storage duration, significantly maximum electrical conductivity was observed in $\mathrm{T}_{0}(0.604$ $\mathrm{ds} / \mathrm{cm} / \mathrm{seed}, 0.634 \mathrm{ds} / \mathrm{cm} / \mathrm{seed}, 0.688 \mathrm{ds} / \mathrm{cm} / \mathrm{seed}, 0.735 \mathrm{ds} / \mathrm{cm} / \mathrm{seed}$ and $0.786 \mathrm{ds} / \mathrm{cm} / \mathrm{seed}$ ) respectively. Maximum electrical conductivity was recorded in G-2 $(0.373 \mathrm{ds} / \mathrm{cm} / \mathrm{seed})$ which was followed by G-3 $(0.299 \mathrm{ds} / \mathrm{cm} / \mathrm{seed})$ and G-1 $(0.283 \mathrm{ds} / \mathrm{cm} / \mathrm{seed})$ during 0 month storage. In 2, 4, 6, 8 and 10 months storage duration, significantly maximum electrical conductivity was observed in G-2 $(0.477 \mathrm{ds} / \mathrm{cm} / \mathrm{seed}, 0.496 \mathrm{ds} / \mathrm{cm} / \mathrm{seed}, 0.534 \mathrm{ds} / \mathrm{cm} / \mathrm{seed}$, $0.573 \mathrm{ds} / \mathrm{cm} / \mathrm{seed}$ and $0.607 \mathrm{ds} / \mathrm{cm} / \mathrm{seed}$ ) respectively.

\section{Introduction}

Floriculture is a branch of horticulture concerned with the cultivation of commercial flowers and ornamental plants for gardens and for floristy. Flowers have been considered as the symbol of grace and elegance and a feast for our eyes. Floriculture is the art and knowledge of growing flowers to perfection. In India, floriculture is not getting the priority it deserves, though it has a great role to play. It is an intensive type of agriculture and the 
income per acre is much higher than any other agricultural product if it is done in a scientific way. Flowers symbolize purity, beauty, peace, love, and passion.

Marigold (Tagetes patula L.), a member of family compositae is an important loose flower crop cultivated in India. It is native to Mexico and commonly called as Jafri and Gaindi. In India, it is mainly grown for commercial purposes in the states of Karnataka, Maharashtra, Haryana, Madhya Pradesh, Gujarat, Andhra Pradesh and Odisha. The marigold is also grown as trap crop to control pest activity. Poultry industry is extensively using marigold petals as a natural source of xanthophylls pigment. Thus, cultivation of marigold gaining popularity day by day in agriculture business owing to its commercial usage.

Marigold seeds exhibit a rapid decline in vigour and viability under ambient conditions of storage from April to October in Northern plains (Rao et al., 2003). Non availability of high quality seeds of marigold is one of the major constraints in its cultivation. Seed possesses maximum vigour at the time of physiological maturity, thereafter in viability and vigour of seed decline gradually.

Thus, the preparation of seed with high vigour is essential to improve seed storability and seedling establishment which ultimately enhance the productivity under wide range of field conditions. Little information has been reported on seedling development of marigold subsequent to priming. Priming treatments have been reported to offer promising means for maintaining the quality of different crop species. Improved seed invigouration techniques are being used in many parts of world to reduce the germination time, synchronize germination, improve germination rate and increase seedling stand (Khan 1992, Lee and Kim, 2000). Various treatments involving hydration-dehydration or pre sowing treatments with different chemicals $-\mathrm{CaCl}_{2}$ and $\mathrm{KNO}_{3}$ for increasing the storage of seeds life have been found beneficial. Therefore, a laboratory study was carried out to assess the influence of various priming treatments on germination and electrical conductivity of French marigold seeds.

\section{Materials and Methods}

A good quality seed is an essential requirement for obtaining higher yields per unit area. To assess the seed quality during storage this research was conducted in laboratory of Seed Science and Technology department, CCS Haryana Agricultural University. The research was planned to determine the ageing, priming and enzyme activity on seed quality parameters of marigold seeds under natural and artificial aged conditions. An effort was made to assess effect of priming treatments on seed quality during storage and the effect of accelerated ageing on primed seed genotypes during storage.

The seed material for the present investigation was collected from Horticulture farm, Department of Horticulture CCS Haryana Agricultural University Hisar. The study was carried out on marigold seeds of four lines which were harvested during April. The harvested seeds of different lines (MGH208, MGH205, MGH207 and Hissar Jafri) were designated as Genotype-1; Genotype-2; Genotype-3; and Genotype-4, respectively.

\section{Treatments}

The seeds of various genotypes (G-1, G-2, G3 and G-4) were harvested in $16^{\text {th }}$ April and after processing the seeds were primed and observations were recorded at two months interval upto February (2017). 
$\mathrm{T}_{0}$ - Untreated (control)

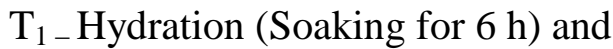
dehydration at room temperature

$\mathrm{T}_{2-} 2 \% \mathrm{CaCl}_{2}$ (Soaking for $6 \mathrm{~h}$ ) and dehydration at room temperature.

$\mathrm{T}_{3-} 4 \% \mathrm{CaCl}_{2}$ (Soaking for $6 \mathrm{~h}$ ) and dehydration at room temperature.

$\mathrm{T}_{4-} 0.5 \% \mathrm{KNO}_{3}$ (Soaking for $6 \mathrm{~h}$ ) and dehydration at room temperature.

$\mathrm{T}_{5-} 1 \% \mathrm{KNO}_{3}$ (Soaking for $6 \mathrm{~h}$ ) and dehydration at room temperature.

$\mathrm{T}_{6-} 2 \%$ Mannitol (Soaking for $6 \mathrm{~h}$ ) and dehydration at room temperature.

$\mathrm{T}_{7--} 4 \%$ Mannitol (Soaking for $6 \mathrm{~h}$ ) and dehydration at room temperature.

$\mathrm{T}_{8--} 6 \%$ Mannitol (Soaking for $6 \mathrm{~h}$ ) and dehydration at room temperature.

Method of application of priming treatments

Sufficient number of seeds from different seed genotypes were placed over filter-paper soaked in solution of the desired treatment in a beaker and kept it at room temperature.

The seeds were allowed to imbibe solution for $6 \mathrm{~h}$ in all the treatments. After the completion of treatment period, the seeds were dehydrated at room temperature.

\section{Standard germination test $(\%)$}

Fifty seeds of each lot replicated thrice were placed on sufficiently in petri plates and placed in seed germinator at $20^{\circ} \mathrm{C}$ temperature with $80-85$ per cent relative humidity. The final count of germinated seeds was taken on 10th day and normal seedlings were expressed as percent germination of total seeds.

\section{Electrical conductivity test (EC) $(\mathrm{ds} / \mathrm{cm} / \mathrm{seed})$}

Twenty normal and uninjured seeds were soaked in separate beakers each containing 50 $\mathrm{ml}$ of distilled water. Seeds were immersed completely in water and beakers were covered with foil. Thereafter, these samples were placed in germinator at $20^{\circ} \mathrm{C}$ for 24 hours in dark. The electrical conductivity of seed leachates was measured by conductivity bridge meter (ISTA, 1999).

\section{Results and Discussion}

\section{Standard germination test (\%)}

The various priming treatments had significant effect on the germination percentage in marigold (Table 1). Maximum germination percentage was recorded in G-4(83.556\%) which was followed by G-1 (79.33\%) and G-3 (76.222\%) during 0 month storage. In 2, 4, 6, 8 and 10 months storage duration, significantly maximum germination percentage was observed in G-4 $(81.741 \%$, $74.444 \%, 65.926 \%, 55.963 \%$ and $43.963 \%$ ) respectively. The highest germination percentage was recorded in $\mathrm{T}_{4}(83.00 \%)$ during 0 month storage. In 2, 4, 6, 8 and 10 months storage duration, significantly maximum germination percentage was observed in $\mathrm{T}_{4}(81.333 \%, 75.333 \%, 69.33 \%$, $60.917 \%$ and $44.250 \%$ ) respectively.

The germination percentage observed in $\mathrm{T}_{\mathbf{4}}$ and G-4 interaction (89.00\%) was non significant in 0 month storage. In 2, 4, 6, 8 and 10 months storage duration, significantly maximum germination percentage was observed in $\mathrm{T}_{4}$ and G-4 interaction $(87.00 \%$, $80.667 \%, 74.667 \%, 68.00 \%$ and $50.33 \%$ ) respectively 
Electrical conductivity test (EC) its role in influencing the permeability of $(\mathrm{ds} / \mathrm{cm} / \mathrm{seed})$ membranes, which ultimately leads to activation of enzymes involved in protein synthesis, carbohydrate metabolism. These results are in line with Nawaz et al., (2011) in tomato seeds, Afzal et al., (2009) in marigold seeds, Abdollahi et al., (2012) in canola seeds and Yam et al., (2015) in Chinese cabbage seeds.

$3 \quad(0.299 \mathrm{ds} / \mathrm{cm} / \mathrm{seed})$ and G-1 $(0.283$ $\mathrm{ds} / \mathrm{cm} / \mathrm{seed}$ ) during 0 month storage. In $2,4,6$, 8 and 10 months storage duration, significantly maximum electrical conductivity was observed in G-2 $(0.477 \mathrm{ds} / \mathrm{cm} / \mathrm{seed}, 0.496$ $\mathrm{ds} / \mathrm{cm} / \mathrm{seed}, \quad 0.534 \mathrm{ds} / \mathrm{cm} / \mathrm{seed}, \quad 0.573$ $\mathrm{ds} / \mathrm{cm} / \mathrm{seed}$ and $0.607 \mathrm{ds} / \mathrm{cm} / \mathrm{seed}$ ) respectively. The highest electrical conductivity was recorded in $\mathrm{T}_{0}(0.499 \mathrm{ds} / \mathrm{cm} / \mathrm{seed})$ during 0 month storage. In 2, 4, 6, 8 and 10 months storage duration, significantly maximum electrical conductivity was observed in $\mathrm{T}_{0}$ $(0.604 \mathrm{ds} / \mathrm{cm} / \mathrm{seed}, 0.634 \mathrm{ds} / \mathrm{cm} / \mathrm{seed}, 0.688$ $\mathrm{ds} / \mathrm{cm} / \mathrm{seed}, \quad 0.735 \mathrm{ds} / \mathrm{cm} / \mathrm{seed}$ and $0.786 \mathrm{ds} / \mathrm{cm} / \mathrm{seed}$ ) respectively.

The highest electrical conductivity was observed in $\mathrm{T}_{\mathbf{0}}$ and $\mathrm{G}-2$ interaction $(0.570$ $\mathrm{ds} / \mathrm{cm} / \mathrm{seed}$ ) during 0 month storage. In $2,4,6$, 8 and 10 months storage duration, significantly maximum electrical conductivity was observed in $\mathrm{T}_{0}$ and G-2 interaction $(0.670$ $\mathrm{ds} / \mathrm{cm} / \mathrm{seed}, \quad 0.687 \mathrm{ds} / \mathrm{cm} / \mathrm{seed}, \quad 0.720$ $\mathrm{ds} / \mathrm{cm} / \mathrm{seed}, \quad 0.750 \mathrm{ds} / \mathrm{cm} / \mathrm{seed} \quad$ and $0.783 \mathrm{ds} / \mathrm{cm} / \mathrm{seed}$ ) respectively

\section{Standard germination}

Effect of priming treatments on standard germination of marigold seeds has been recorded in the Table 1. At 0 month storage period treatment $\mathrm{T}_{4} \quad\left(\begin{array}{llll}0.5 \% & \left.\mathrm{KNO}_{3}\right) & \text { and }\end{array}\right.$ genotype G-4 was highly significant compared to all other treatments and genotypes. For 2, 4, 6, 8and 10 month storage, $\mathrm{T}_{4}$ and G-4 produced maximum germination percentage. The positive effect of $\mathrm{KNO}_{3}$ might be due to

\section{Electrical conductivity}

Electrical conductivity in the marigold seed of different priming treatment for 0 month storage was found highest in the treatment $\mathrm{T}_{0}$ (control) compared to all other treatments. Among different genotypes G-2 was highly significant as compared to other genotypes as presented in the Table 2. Similar results were observed over the other storage duration. This might be due to destructive changes in cellular membrane system resulting in more leakage of organic solutes (free sugars, fatty acids and amino acids). Damage to membrane system could be repaired and protected against such changes by invigouration treatments with $\mathrm{KNO}_{3}$ as evidenced by low electrical conductivity of seed leachates, which presumably have extended the viability of seeds. The differential EC values recorded among the seed treatments indicate the nature and extent of membrane protection offered, which may not be the same for all seed priming treatments, thus resulting in difference EC values.

Fresh seeds were tested for various vigour and viability parameters in a completely randomized design with three replications.

The statistical analysis of data for various parameters was carried out according to the standard procedure. The results obtained during the investigation are summarized as below: 
Table.1 Effect of priming treatments on germination of marigold seeds

\begin{tabular}{|c|c|c|c|c|c|c|c|c|c|c|c|}
\hline \multirow{3}{*}{$\begin{array}{c}\begin{array}{c}\text { Storage } \\
\text { duration }\end{array} \\
\text { In months }\end{array}$} & \multirow{3}{*}{$\begin{array}{c}\text { Seed } \\
\text { genotypes }\end{array}$} & \multicolumn{9}{|c|}{ Germination percentage (\%) } & \multirow{3}{*}{$\begin{array}{c}\text { Mean } \\
(\%)\end{array}$} \\
\hline & & \multicolumn{9}{|c|}{ Priming treatments } & \\
\hline & & $\mathbf{T}_{0}$ & $T_{1}$ & $\mathbf{T}_{2}$ & $\mathbf{T}_{3}$ & $\mathbf{T}_{4}$ & $\mathbf{T}_{5}$ & $T_{6}$ & $\mathbf{T}_{7}$ & T8 & \\
\hline \multirow[t]{4}{*}{ 0 } & G-1 & 82.000 & 79.333 & 80.000 & 80.333 & 87.000 & 82.000 & 77.000 & 74.000 & 72.333 & 79.333 \\
\hline & G-2 & 77.000 & 73.000 & 72.000 & 73.000 & 75.000 & 73.000 & 72.000 & 69.000 & 69.000 & 72.556 \\
\hline & G-3 & 79.000 & 76.000 & 75.000 & 78.000 & 81.000 & 76.000 & 74.000 & 71.000 & 76.000 & 76.222 \\
\hline & G-4 & 87.000 & 82.000 & 82.000 & 85.000 & 89.000 & 87.000 & 82.000 & 79.000 & 79.000 & 83.556 \\
\hline \multicolumn{2}{|c|}{ Mean } & 81.250 & 77.583 & 77.250 & 79.083 & 83.000 & 79.500 & 76.250 & 73.250 & 74.083 & \\
\hline \multirow[t]{4}{*}{2} & G-1 & 80.000 & 77.333 & 78.333 & 77.000 & 85.667 & 80.333 & 75.000 & 72.667 & 70.333 & 77.407 \\
\hline & G-2 & 75.000 & 71.000 & 70.333 & 71.667 & 73.333 & 71.000 & 70.000 & 67.000 & 67.667 & 70.778 \\
\hline & G-3 & 77.667 & 74.667 & 73.000 & 76.333 & 79.333 & 74.333 & 72.000 & 69.667 & 74.333 & 74.593 \\
\hline & G-4 & 85.000 & 80.000 & 80.000 & 83.000 & 87.000 & 85.333 & 80.000 & 77.667 & 77.667 & 81.741 \\
\hline \multicolumn{2}{|c|}{ Mean } & 79.417 & 75.750 & 75.417 & 77.000 & 81.333 & 77.750 & 74.250 & 71.750 & 72.500 & \\
\hline \multirow[t]{4}{*}{4} & G-1 & 72.000 & 66.000 & 70.667 & 73.000 & 77.000 & 74.000 & 70.000 & 65.667 & 65.000 & 70.370 \\
\hline & G-2 & 63.000 & 63.667 & 65.000 & 64.333 & 70.000 & 65.333 & 65.000 & 59.667 & 63.000 & 64.333 \\
\hline & G-3 & 65.333 & 64.667 & 65.000 & 69.667 & 73.667 & 68.333 & 66.000 & 62.000 & 65.333 & 66.667 \\
\hline & G-4 & 76.667 & 70.333 & 74.333 & 76.333 & 80.667 & 77.333 & 73.000 & 72.000 & 69.333 & 74.444 \\
\hline \multicolumn{2}{|c|}{ Mean } & 69.250 & 66.167 & 68.750 & 70.833 & 75.333 & 71.250 & 68.500 & 64.833 & 65.667 & \\
\hline \multirow[t]{4}{*}{6} & G-1 & 62.667 & 60.000 & 62.333 & 66.667 & 69.000 & 65.000 & 60.667 & 57.000 & 57.333 & 62.296 \\
\hline & G-2 & 56.000 & 52.000 & 55.667 & 56.000 & 62.000 & 55.667 & 53.333 & 49.667 & 52.000 & 54.704 \\
\hline & G-3 & 59.000 & 58.000 & 48.333 & 63.667 & 71.667 & 62.333 & 59.667 & 53.000 & 55.667 & 59.037 \\
\hline & G-4 & 66.000 & 61.333 & 64.333 & 68.667 & 74.667 & 68.667 & 66.333 & 62.333 & 61.000 & 65.926 \\
\hline \multicolumn{2}{|c|}{ Mean } & 60.917 & 57.833 & 57.667 & 63.750 & 69.333 & 62.917 & 60.000 & 55.500 & 56.500 & \\
\hline \multirow[t]{4}{*}{8} & G-1 & 49.333 & 46.333 & 55.000 & 58.667 & 59.667 & 56.333 & 45.333 & 41.333 & 46.000 & 50.889 \\
\hline & G-2 & 44.333 & 40.333 & 40.333 & 41.667 & 54.333 & 48.667 & 40.667 & 36.000 & 34.667 & 42.333 \\
\hline & G-3 & 49.000 & 44.000 & 43.000 & 54.333 & 61.667 & 50.000 & 42.000 & 39.667 & 36.000 & 46.630 \\
\hline & G-4 & 53.333 & 50.000 & 57.333 & 60.000 & 68.000 & 57.333 & 57.667 & 50.333 & 49.667 & 55.963 \\
\hline \multicolumn{2}{|c|}{ Mean } & 49.000 & 45.167 & 48.917 & 53.667 & 60.917 & 53.083 & 46.417 & 41.833 & 41.583 & \\
\hline \multirow[t]{4}{*}{10} & G1 & 38.667 & 38.333 & 39.333 & 45.333 & 46.333 & 43.000 & 41.000 & 38.000 & 36.000 & 40.667 \\
\hline & G-2 & 33.333 & 31.667 & 33.667 & 36.000 & 36.333 & 34.333 & 33.333 & 32.333 & 31.667 & 33.630 \\
\hline & G-3 & 34.333 & 32.333 & 39.667 & 40.667 & 44.000 & 39.333 & 38.000 & 36.000 & 34.667 & 37.667 \\
\hline & G-4 & 41.333 & 39.667 & 44.333 & 47.333 & 50.333 & 49.333 & 44.667 & 40.333 & 38.333 & 43.963 \\
\hline \multicolumn{2}{|c|}{ Mean } & 36.917 & 35.500 & 39.250 & 42.333 & 44.250 & 41.500 & 39.250 & 36.667 & 35.167 & \\
\hline \multicolumn{12}{|c|}{ C.D. at $5 \%$ Seed } \\
\hline \multicolumn{3}{|c|}{ Genotype $=1.464$} & entis & 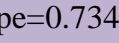 & & & & enotyn & & & \\
\hline \multirow{2}{*}{\multicolumn{3}{|c|}{$\begin{array}{l}\text { Treatment }=2.196 \\
\text { Genotype X Treatments }=\text { NS }\end{array}$}} & Treatn & ents $=0.10$ & & & & Treatment & $=0.925$ & & \\
\hline & & & Genot & pe X Tre & atments $=$ & .203 & & Genotype & $\mathrm{x}$ Treatm & $\mathrm{nts}=1.85 \mathrm{C}$ & \\
\hline \multicolumn{3}{|c|}{ Genotype $=0.662$} & \multicolumn{5}{|c|}{ Genotype $=0.879$} & \multicolumn{3}{|c|}{ Genotype $=0.866$ Treatment } & 1.993 \\
\hline Treatments & $=1.318$ & & Treatn & ents $=1.2$ & & & & Genotype & $X$ Treatm & ents $=2.9$ & \\
\hline Genotype & Treatments & & Genot & pe X Tre & tments = & & & & & & \\
\hline
\end{tabular}


Table.2 Effect of priming treatments on Electrical conductivity of marigold seeds

\begin{tabular}{|c|c|c|c|c|c|c|c|c|c|c|c|}
\hline \multirow{3}{*}{$\begin{array}{c}\begin{array}{c}\text { Storage } \\
\text { duration }\end{array} \\
\text { In months }\end{array}$} & \multirow{3}{*}{$\begin{array}{c}\text { Seed } \\
\text { genotype }\end{array}$} & \multicolumn{9}{|c|}{ Electrical conductivity $(\mathrm{ds} / \mathrm{cm} / \mathrm{seed})$} & \multirow[t]{3}{*}{ Mean } \\
\hline & & \multicolumn{9}{|c|}{ Priming treatments } & \\
\hline & & $\mathbf{T}_{0}$ & $\mathbf{T}_{1}$ & $\mathbf{T}_{2}$ & $\mathbf{T}_{3}$ & $\mathbf{T}_{4}$ & $\mathbf{T}_{5}$ & $\mathbf{T}_{6}$ & $\mathbf{T}_{7}$ & T8 & \\
\hline \multirow[t]{4}{*}{ 0 } & G-1 & 0.434 & 0.280 & 0.330 & 0.360 & 0.240 & 0.270 & 0.230 & 0.200 & 0.200 & 0.283 \\
\hline & G-2 & 0.570 & 0.330 & 0.470 & 0.440 & 0.330 & 0.310 & 0.310 & 0.310 & 0.290 & 0.373 \\
\hline & G-3 & 0.510 & 0.300 & 0.320 & 0.320 & 0.300 & 0.300 & 0.170 & 0.370 & 0.100 & 0.299 \\
\hline & G-4 & 0.480 & 0.360 & 0.350 & 0.377 & 0.270 & 0.250 & 0.220 & 0.210 & 0.210 & 0.303 \\
\hline \multicolumn{2}{|c|}{ Mean } & 0.499 & 0.318 & 0.368 & 0.374 & 0.285 & 0.283 & 0.233 & 0.273 & 0.200 & \\
\hline \multirow[t]{4}{*}{2} & G-1 & 0.545 & 0.387 & 0.430 & 0.457 & 0.353 & 0.377 & 0.307 & 0.307 & 0.303 & 0.385 \\
\hline & G-2 & 0.670 & 0.440 & 0.573 & 0.557 & 0.433 & 0.417 & 0.417 & 0.407 & 0.380 & 0.477 \\
\hline & G-3 & 0.613 & 0.303 & 0.413 & 0.427 & 0.403 & 0.407 & 0.287 & 0.270 & 0.203 & 0.370 \\
\hline & G-4 & 0.587 & 0.350 & 0.453 & 0.480 & 0.370 & 0.357 & 0.330 & 0.307 & 0.307 & 0.393 \\
\hline \multicolumn{2}{|c|}{ Mean } & 0.604 & 0.370 & 0.468 & 0.480 & 0.390 & 0.389 & 0.335 & 0.323 & 0.298 & \\
\hline \multirow[t]{4}{*}{4} & G-1 & 0.620 & 0.420 & 0.463 & 0.487 & 0.373 & 0.407 & 0.330 & 0.313 & 0.337 & 0.417 \\
\hline & G-2 & 0.687 & 0.470 & 0.600 & 0.587 & 0.480 & 0.457 & 0.430 & 0.410 & 0.347 & 0.496 \\
\hline & G-3 & 0.610 & 0.330 & 0.450 & 0.470 & 0.430 & 0.430 & 0.323 & 0.313 & 0.330 & 0.410 \\
\hline & G-4 & 0.620 & 0.390 & 0.480 & 0.520 & 0.390 & 0.370 & 0.353 & 0.330 & 0.330 & 0.420 \\
\hline \multicolumn{2}{|c|}{ Mean } & 0.634 & 0.403 & 0.498 & 0.516 & 0.418 & 0.416 & 0.359 & 0.342 & 0.336 & \\
\hline \multirow[t]{4}{*}{6} & G-1 & 0.680 & 0.440 & 0.500 & 0.510 & 0.410 & 0.430 & 0.380 & 0.340 & 0.343 & 0.448 \\
\hline & G-2 & 0.720 & 0.517 & 0.620 & 0.640 & 0.503 & 0.490 & 0.480 & 0.450 & 0.390 & 0.534 \\
\hline & G-3 & 0.650 & 0.373 & 0.490 & 0.490 & 0.450 & 0.453 & 0.353 & 0.350 & 0.320 & 0.437 \\
\hline & G-4 & 0.703 & 0.470 & 0.510 & 0.510 & 0.410 & 0.400 & 0.390 & 0.370 & 0.350 & 0.457 \\
\hline \multicolumn{2}{|c|}{ Mean } & 0.688 & 0.450 & 0.530 & 0.538 & 0.443 & 0.443 & 0.401 & 0.378 & 0.351 & \\
\hline \multirow[t]{4}{*}{8} & G-1 & 0.710 & 0.470 & 0.530 & 0.560 & 0.460 & 0.473 & 0.410 & 0.380 & 0.370 & 0.485 \\
\hline & G-2 & 0.750 & 0.490 & 0.670 & 0.710 & 0.513 & 0.530 & 0.543 & 0.520 & 0.430 & 0.573 \\
\hline & G-3 & 0.690 & 0.390 & 0.520 & 0.570 & 0.477 & 0.460 & 0.390 & 0.390 & 0.353 & 0.471 \\
\hline & G-4 & 0.790 & 0.500 & 0.550 & 0.600 & 0.490 & 0.473 & 0.430 & 0.410 & 0.390 & 0.515 \\
\hline \multicolumn{2}{|c|}{ Mean } & 0.735 & 0.463 & 0.567 & 0.610 & 0.485 & 0.484 & 0.443 & 0.425 & 0.386 & \\
\hline \multirow[t]{4}{*}{10} & G-1 & 0.750 & 0.490 & 0.560 & 0.593 & 0.480 & 0.490 & 0.430 & 0.410 & 0.400 & 0.511 \\
\hline & G-2 & 0.783 & 0.507 & 0.690 & 0.733 & 0.590 & 0.570 & 0.573 & 0.550 & 0.467 & 0.607 \\
\hline & G-3 & 0.730 & 0.427 & 0.550 & 0.610 & 0.500 & 0.490 & 0.413 & 0.420 & 0.390 & 0.503 \\
\hline & G-4 & 0.880 & 0.590 & 0.610 & 0.670 & 0.530 & 0.523 & 0.490 & 0.473 & 0.450 & 0.580 \\
\hline \multicolumn{2}{|c|}{ Mean } & 0.786 & 0.503 & 0.603 & 0.652 & 0.525 & 0.518 & 0.477 & 0.463 & 0.427 & \\
\hline \multicolumn{4}{|c|}{ C.D. at $5 \%$ Genotype $=0.012$} & Genotype & & & \multicolumn{4}{|c|}{ Genotype $=0.005$} & \\
\hline \multirow{3}{*}{\multicolumn{4}{|c|}{$\begin{array}{l}\text { Treatment } \quad=0.018 \\
\text { Genotype X Treatments }=0.035 \\
\text { Genotype }=0.007\end{array}$}} & Treatme & $=0.008$ & & \multicolumn{4}{|c|}{ Treatments $=0.008$} & \\
\hline & & & & Genotyp & $X$ Treat & nts $=0.0$ & \multicolumn{4}{|c|}{ Genotype X Treatments $=0.016$} & \\
\hline & & & & Genotyl & $=0.010$ & & \multicolumn{4}{|c|}{ Genotype $=0.009$} & \\
\hline \multicolumn{4}{|c|}{ Treatment $=0.011$} & Treatme & $=0.015$ & & & atments & 0.014 & & \\
\hline Genotype $Y$ & reatments $=0$ & & & Genotyp & K Treatr & ts $=0.03$ & & hotype 2 & Treatme & $=0.028$ & \\
\hline
\end{tabular}

Seeds were primed with various priming treatments viz., 0.5 and $2 \% \mathrm{KNO}_{3}, 2$ and $4 \%$ $\mathrm{CaCl}_{2}, 2$, 4 and 6\% Mannitol, control and dehydration. Treatment $\mathrm{T}_{4} \quad\left(\begin{array}{lll}0.5 \% & \mathrm{KNO}_{3}\end{array}\right)$ exhibited higher germination as compared to other treatments and Seed genotype-4 was found to be performing significantly better germination than rest of genotypes and The highest electrical conductivity was observed in $\mathrm{T}_{\mathbf{0}}$ and $\mathrm{G}-2$ interaction

\section{References}

Abdollahi, Farzin and Jafari., 2012. Effect of $\mathrm{NaCl}$ and $\mathrm{KNO}_{3}$ priming on seed germination of canola (Brassica napus L.) under salinity conditions. International J. Agriculture: Research and Review, 2(5):573-579.

Afzal, I., Ashraf, S., Qasim, M., Basra, S.M.A. and Shahid, M., 2009. Does halopriming improve germination and 
seedling vigour in marigold (Tagetes spp.). Seed Science and Technology, 37(2): 436-445.

Dong, L., Hao, Z., Li, Z., Zhu, J. and Wang, Q., 2014. Enhancement of Welsh Onion (Allium fistulosum L.) Seed vigour by $\mathrm{KNO}_{3}$ priming. Journal of Agricultural Science and Technology, 16(6):1345-1353.

ISTA, 1999. International rules for seed testing. Seed Science and Technology, 23(Suppl.): 1-334.

Jegathambal, R. and Shanmugam, K., 2007. Pre-sowing seed treatment to improve germination in true cassava (Manihot esculenta) seeds. Madras Agricultural Journal, 94(7/12): 189-191.

Khan, A.A. 1992. Preplant physiological seed conditioning. Horticultural Reviews, 14: $131-181$
Lee, S.S. and Kim, J.H.2000. Total sugars, amylase activity and germination after priming rice seeds. Korean Journal of Crop Science, 44(2): 108-111.

Nawaz, A., Amjad, M., Pervez, M.A. and Afzal, I., 2011. Effect of halopriming on germination and seedling vigor of tomato. African Journal of Agricultural Research, 6(15): 35513559.

Rao,C.C., Dadlani, N.K. and Dadlani, M. 2003. Maintenance and enhancement of germination and vigour in marigold (Tagetes spp.) Seed Science and Technology, 32(2): 503-509.

Yan, M., 2015. Seed priming stimulate germination and early seedling growth of Chinese cabbage under drought stress. South African Journal of Botany, 99: 88-92.

\section{How to cite this article:}

Sindhu, K. and Sehrawat, S.K. 2019. Effect of Seed Priming on Standard Germination and Electrical Conductivity in Marigold Seeds. Int.J.Curr.Microbiol.App.Sci. 8(11): 1686-1692. doi: https://doi.org/10.20546/ijcmas.2019.811.196 